\title{
Two-Layer Flow with One Viscous Layer in Inclined Channels
}

\author{
O.K. Matar ${ }^{a 1}$, G.M. Sisoev ${ }^{b}$ and C.J. Lawrence ${ }^{c}$ \\ ${ }^{a}$ Department of Chemical Engineering, Imperial College London \\ South Kensington Campus, SW7 2AZ, UK \\ ${ }^{b}$ School of Mathematics, University of Birmingham, Edgbaston \\ Birmingham, B15 2TT, UK \\ ${ }^{c}$ Institutt for Energiteknikk, P.O. Box 40, 2027 Kjeller, Norway
}

\begin{abstract}
We study pressure-driven, two-layer flow in inclined channels with high density and viscosity contrasts. We use a combination of asymptotic reduction, boundary-layer theory and the Karman-Polhausen approximation to derive evolution equations that describe the interfacial dynamics. Two distinguished limits are considered: where the viscosity ratio is small with density ratios of order unity, and where both density and viscosity ratios are small. The evolution equations account for the presence of inertia, gravity, capillarity and viscous retardation; attention is restricted to situations in which the flow is laminar. The results of our linear stability analysis and numerical simulations indicate that the flow is destabilised by positive channel inclination in the stably stratified case. The dependence of the nonlinear wave dynamics on system parameters is also explored.
\end{abstract}

Key words: slug flows, interfacial instability, two-layer flow, channel flow, modelling AMS subject classification: 35Q30, 35Q35, 76D05, 76D08, 76D33, 76D45, 76T10

\section{Introduction}

Flows involving either gas-liquid or liquid-liquid systems are of central importance to the design and operation of process equipment in the oil and gas industry. It is therefore not surprising that these flows have received considerable attention in the literature. Previous studies have focused on

\footnotetext{
${ }^{1}$ Corresponding author. E-mail: : o.matar@imperial.ac.uk
} 
predicting transitions from stratified to "slug" flows in gas-liquid systems [1]-[3]. Linear stability analyses, involving the solution of two-layer Orr-Sommerfeld problems or long-wave theory have been carried out [2], [4]-[10]. The dynamics in the nonlinear regime have also been probed using one-dimensional averaged equations [11,12] and direct numerical simulations [13]-[16]. Previous work has also examined the dynamics of multi-layer flows in the context of industrial coating flows and geophysical flows $[17,18]$ and focused on situations wherein the upper interface is a free surface.

Long-wave theory has been used extensively in the literature to model the dynamics of interfacial waves. Studies based on this theory exploit the small aspect ratios of certain wave regimes that emerge during the flow to derive an evolution equation for the interfacial position. Tilley et al. [19] (see also [20]) used this approach to derive a highly nonlinear evolution equation for the interface separating immiscible, viscous layers in an inclined channel. From this equation, these authors then derived a generalised Kuramoto-Sivashinsky equation (KSE) and, in the limit of small advection contributions, a modified KSE; this equation possesses a cubic nonlinearity due to the two-phase nature of the flow. Through the use of weakly nonlinear and bifurcation analyses, the modified KSE was shown to exhibit hysteretic behaviour that manifested itself as jumps from small- to large-amplitude travelling waves; this behaviour is absent in KS dynamics and was interpreted as a precursor to "flooding" in gas-liquid flows.

More recently, Segin et al. [21,22] modelled situations relevant to counter-current gas-liquid flows with either fixed gas flow rate or fixed liquid flow rate and pressure drop. They used lubrication theory to derive evolution equations for the interface shape and pressure and show the development of "Lax" and "undercompressive" shocks, and rarefaction waves for different system parameters. Matar et al. [23] considered the pressure-driven channel flow of two viscous, immiscible, density-matched fluids in the context of cleaning-type applications with large viscosity contrasts. They used long-wave theory together with the Karman-Polhausen approximation to derive a single evolution equation for the thickness of the more viscous layer; this equation was shown to reduce to a modified Benney equation and the KSE for sufficiently small mean film thicknesses. Matar et al. used bifurcation theory and numerical simulations to show the development of large-amplitude interfacial waves, which for sufficiently large mean film thicknesses and/or large inertial contributions led to channel bridging; this is analogous to slug-formation in gas-liquid flows. This study, however, neglected the presence of density variations and gravitational effects, which can play an important role in the dynamics.

In this paper, we revisit the analysis of Matar et al. [23] and account for gravitational effects by considering inclined channels and density variations. We study the flow of a fast-flowing layer of one fluid past a layer of a highly viscous fluid and examine situations in which the ratio of the upper to lower fluid density is either of order unity or very low. The former and latter cases are representative of cleaning flows involving liquid-liquid systems, and gas-liquid flows, respectively. The dynamics are described in the first case by a single evolution equation for the interface, and in the second case by a two-equation system, with an additional evolution equation for the flow rate in the more viscous layer.

The rest of this paper is organised as follows. In Section 2, we provide details of the derivation of the evolution equations. A discussion of our analytical and numerical results is presented in 
Section 3. Finally, concluding remarks are provided in Section 4.

\section{Problem formulation}

We consider pressure-driven two-layer flow in a channel of height $H$ inclined at an angle $\theta$ measured upwards from the horizontal. Both fluids are considered Newtonian, immiscible and incompressible and the channels walls are assumed to be rigid and impermeable. The upper (lower) fluid has density and viscosity $\rho_{U}$ and $\mu_{U}\left(\rho_{L}\right.$ and $\left.\mu_{L}\right) ; U$ and $L$ are used to designate the properties of the upper and lower fluids, respectively. The two-dimensional dynamics are modelled using rectangular coordinates $(x, z)$ where $x$ and $z$ denote the directions parallel and normal to the wall, respectively. The upper and lower fluids are located in $h \leq z \leq H$ and $0 \leq z \leq h$, respectively, separated by an interface at $z=h(x, t)$ endowed with a constant interfacial tension, $\gamma$.

\subsection{Governing equations and scaling}

In each layer, the governing equations correspond to the two-dimensional mass conservation and Navier-Stokes equations:

$$
\begin{gathered}
U_{x}+W_{z}=0, \\
\rho_{U}\left(U_{t}+U U_{x}+W U_{z}\right)=-\rho_{U} g \sin \theta-P_{x}+\mu_{U}\left(U_{x x}+U_{z z}\right), \\
\rho_{U}\left(W_{t}+U W_{x}+W W_{z}\right)=-\rho_{U} g \cos \theta-P_{z}+\mu_{U}\left(W_{x x}+W_{z z}\right), \\
u_{x}+w_{z}=0, \\
\rho_{L}\left(u_{t}+u u_{x}+w u_{z}\right)=-\rho_{L} g \sin \theta-p_{x}+\mu_{L}\left(u_{x x}+u_{z z}\right), \\
\rho_{L}\left(w_{t}+u w_{x}+w w_{z}\right)=-\rho_{L} g \cos \theta-p_{z}+\mu_{L}\left(w_{x x}+w_{z z}\right),
\end{gathered}
$$

where $U, W$ and $P$ denote the $x$ - and $z$-components of the velocity field, and pressure in the upper fluid, respectively; the analogous variables in the lower layer are given by their lower case counterparts in Eqs. (2..4)-(2..6); $g$ and $t$ represent the gravitational acceleration and time, respectively. The subscripts $x, z$ and $t$ denote partial differentiation with respect to $x, z$ and $t$, respectively.

At the interface, $z=h(x, t)$, we impose appropriate kinematic and dynamic conditions:

$$
\begin{gathered}
u=U, \quad w=W, \quad w-h_{t}-u h_{x}, \\
\mu_{U}\left(\left(U_{z}+W_{x}\right)\left(1-h_{x}^{2}\right)-2 h_{x}\left(U_{x}-W_{z}\right)\right)=\mu_{L}\left(\left(u_{z}+w_{x}\right)\left(1-h_{x}^{2}\right)-2 h_{x}\left(u_{x}-w_{z}\right)\right),
\end{gathered}
$$




$$
\begin{gathered}
P-p-\frac{2 \mu_{U}}{1+h_{x}^{2}}\left(W_{z}+h_{x}^{2} U_{x}-h_{x}\left(U_{z}+W_{x}\right)\right)+\frac{2 \mu_{L}}{1+h_{x}^{2}}\left(w_{z}+h_{x}^{2} u_{x}-h_{x}\left(u_{z}+w_{x}\right)\right)= \\
\frac{\gamma h_{x x}}{\left(1+h_{x}^{2}\right)^{3 / 2}} .
\end{gathered}
$$

At the upper and lower walls, we impose the no-slip and no-penetration conditions:

$$
\begin{gathered}
z=0: \quad u=0, \quad w=0, \\
z=H: \quad U=0, \quad W=0 .
\end{gathered}
$$

The above equations and boundary conditions are rendered dimensionless using the following scaling:

$$
\begin{gathered}
x=L \tilde{x}, \quad(z, h)=H(\tilde{z}, \tilde{h}), \quad t=(L / V) \tilde{t}, \\
(u, U)=V(\tilde{u}, \tilde{U}), \quad(w, W)=\delta V(\tilde{w}, \tilde{W}), \quad(p, P)=\left(\frac{\mu_{U} V L}{H^{2}}\right)(\tilde{p}, \tilde{P}),
\end{gathered}
$$

where the tildes denote the dimensionless variables and the long wave parameter is expressed by $\delta \equiv H / L \ll 1$. Here, $V$ is defined as the superficial velocity in the upper layer, assumed to be constant; the appropriate choice of the length scale $L$ will be discussed below. The dimensionless equations are then given by (following the suppression of the tildes)

$$
\begin{gathered}
U_{x}+W_{z}=0, \\
\delta R e\left(U_{t}+U U_{x}+W U_{z}\right)=-G \sin \theta-P_{x}+\delta^{2} U_{x x}+U_{z z}, \\
\delta^{3} R e\left(W_{t}+U W_{x}+W W_{z}\right)=-G \delta \cos \theta-P_{z}+\delta^{2}\left(W_{z z}+\delta^{2} W_{x x}\right), \\
u_{x}+w_{z}=0, \\
\delta \frac{m}{n} \operatorname{Re}\left(u_{t}+u u_{x}+w u_{z}\right)=-\frac{m}{n} G \sin \theta-m p_{x}+\delta^{2} u_{x x}+u_{z z}, \\
\delta^{3} \operatorname{Re}\left(w_{t}+u w_{x}+w w_{z}\right)=-\frac{m}{n} G \delta \cos \theta-m p_{z}+\delta^{2}\left(w_{z z}+\delta^{2} w_{x x}\right) .
\end{gathered}
$$

The dimensionless interfacial conditions are expressed by

$$
\begin{gathered}
u=U, w=W, w-h_{t}-u h_{x}=0, \\
m\left[\left(U_{z}+\delta^{2} W_{x}\right)\left(1-\delta^{2} h_{x}^{2}\right)-2 \delta^{2} h_{x}\left(U_{x}-W_{z}\right)\right]=\left(u_{z}+\delta^{2} w_{x}\right)\left(1-\delta^{2} h_{x}^{2}\right)-2 \delta^{2} h_{x}\left(u_{x}-w_{z}\right), \quad(2 . .21) \\
P-p-\frac{2 \delta^{2}}{1+\delta^{2} h_{x}^{2}}\left[W_{z}+\delta^{2} h_{x}^{2} U_{x}-h_{x}\left(U_{z}+\delta^{2} W_{x}\right)\right]+\frac{\delta^{2}}{m} \frac{2}{1+\delta^{2} h_{x}^{2}}\left[w_{z}+\delta^{2} h_{x}^{2} u_{x}-h_{x}\left(u_{z}+\delta^{2} w_{x}\right)\right]
\end{gathered}
$$




$$
=\delta^{3} \frac{h_{x x}}{C a\left(1+\delta^{2} h_{x}^{2}\right)^{3 / 2}},
$$

while the boundary conditions at the walls remain unchanged from Eqs. (2..10) and (2..11) (except that the upper wall is now at $z=1$ ). Here the dimensionless parameters are the inclination angle, $\theta$, the viscosity ratio, $m$, and density ratio, $n$, a Reynolds number for the upper layer $R e$, a Capillary number, $C a$, and a gravitational parameter, $G$ :

$$
m \equiv \frac{\mu_{U}}{\mu_{L}}, n \equiv \frac{\rho_{U}}{\rho_{L}}, R e \equiv \frac{\rho_{U} V H}{\mu_{U}}, C a \equiv \frac{\mu_{U} V}{\gamma}, \quad G \equiv \frac{\rho_{U} g H^{2}}{\mu_{U} V}
$$

\subsection{The first distinguished limit: $m \sim \delta, n=O(1)$}

Here, we consider the case of small viscosity ratios and density ratios of order unity, characterized by the distinguished limit, $m \sim \delta$ and $n=O(1)$. We therefore introduce the following re-scalings

$$
R e=\frac{\overline{R e}}{\delta}, \quad C a=\delta^{3} \overline{C a}, \quad(u, w)=m(\bar{u}, \bar{w}), \quad t=\frac{\bar{t}}{m},
$$

where the length scale is chosen as $L \equiv H / m$ and drop terms of $O(\delta)$ to obtain the reduced set of equations

$$
\begin{gathered}
U_{x}+W_{z}=0, \quad \overline{R e}\left(U U_{x}=W U_{z}\right)=-G \sin \theta-P_{x}+U_{x x}, \quad P_{z}=-\delta G \cos \theta, \\
\bar{u}_{x}+\bar{w}_{z}=0, \quad \rho_{x}=-\frac{G \sin \theta}{n}+\bar{u}_{x x}, \quad \rho_{z}=-\frac{1}{n} \delta G \cos \theta
\end{gathered}
$$

and interfacial conditions

$$
U=W=0, \quad \bar{w}-h_{\bar{t}}-\bar{u} h_{x}=0, \quad U_{z}-\bar{u}_{z} \equiv \tau_{i}, \quad P-p=\frac{h_{x x}}{\overline{C a}} .
$$

The wall conditions remain unchanged from Eqs. (2..10) and (2..11).

The mass conservation equations and the momentum equation in the upper fluid are each integrated through the respective layer to obtain

$$
\begin{gathered}
\int_{h}^{1} U d z=Q=Q_{0} \equiv 1, \\
\overline{R e}\left(\int_{h}^{1} U^{2} d z\right)_{x}+(1-h)\left(P_{x}+G \sin \theta\right)+\tau_{w}+\tau_{i}=0, \\
h_{t}+q_{x}=0,
\end{gathered}
$$

where $\left.\tau_{i} \equiv U_{z}\right|_{h}, \tau_{w} \equiv-\left.U_{z}\right|_{l}$ and $q=\int_{0}^{h} \bar{u} d z$ is the flow rate in the more viscous layer. The velocity in the lower layer, $\bar{u}$, which satisfies the interfacial and wall conditions is given by

$$
\bar{u}=\left(p_{x}+\frac{G}{n} \sin \theta\right)\left(\frac{z^{2}}{2}-z h\right)+\tau_{i} z .
$$


The pressure gradient in the upper layer is given by

$$
P_{x}=\left(\left.p\right|_{h}\right)_{x}+\delta G \cos \theta h_{x}+\frac{h_{x x x}}{\overline{C a}} .
$$

Making use of the following closure relation for $U$, which satisfies the boundary conditions at $z=h$ and $z=1$, as well as the flow constraint,

$$
U=-\frac{6}{(1-h)^{3}}\left[z^{2}-z(1+h)+h\right]
$$

yields the following expression for $P_{x}$ from Eq. (2..29)

$$
P_{x}=-G \sin \theta-\frac{6}{5(1-h)^{3}}\left(10+\overline{R e} h_{x}\right) .
$$

Thus, an expression for the interfacial pressure gradient, $\left(\left.p\right|_{h}\right)_{x}$, can be obtained

$$
\left(\left.p\right|_{h}\right)_{x}=-G\left(\sin \theta+\delta h_{x} \cos \theta\right)-\frac{6}{5(1-h)^{3}}\left(10+\overline{R e} h_{x}\right)-\frac{h_{x x x}}{\overline{C a}}
$$

from which the pressure gradient in the lower layer is then given by

$$
\begin{gathered}
p_{x}=\left(\left.p\right|_{h}\right)_{x}+\frac{1}{n} \delta G \cos \theta h_{x} \\
=-\frac{6}{5(1-h)^{3}}\left(10+\overline{R e} h_{x}\right)-\frac{h_{x x x}}{\overline{C a}}+G\left[-\sin \theta+\delta \cos \theta\left(\frac{1}{n}-1\right) h_{x}\right] .
\end{gathered}
$$

Substitution of Eq. (2..35) into Eq. (2..31) and the resultant equation into Eq. (2..30) finally yields an evolution equation for the interfacial position after the introduction of the following re-scalings, $(x, \bar{t})=(3 \overline{C a})^{-1 / 3}(\hat{x}, \hat{t})$ :

$$
h_{t}+\left[\frac{S}{(1-h)^{3}} h^{3} h_{x}+\frac{h^{2}(3+h)}{(1-h)^{3}}-\frac{\bar{G}}{3} h^{3}\left(\sin \theta+\Delta \cos \theta h_{x}\right)+h^{3} h_{x x x}\right]_{x}=0 .
$$

Here, $S \equiv \frac{2}{5} \overline{R e}(3 \overline{C a})^{1 / 3}, \bar{G} \equiv G\left(\frac{1}{n}-1\right)$ and $\Delta \equiv \delta(3 \overline{C a})^{1 / 3}=(3 \overline{C a})^{1 / 3}$ and and the carets have been suppressed.

\subsection{The second distinguished limit: $m \sim \delta^{1 / 2}, n \sim \delta$}

Here, we consider the case of small viscosity and density ratios, characterized by the distinguished limit $m \sim \delta^{1 / 2}$ and $n \sim \delta$; this is appropriate for gas-liquid systems. We introduce Eq. (2..24) along with $m=\bar{m} \delta^{1 / 2}$ and $n=\delta$ into Eqs. (2..14)-(2..22) in order to obtain the following leading order equations, where the length scale is chosen as $L \equiv H / n$

$$
U_{x}+W_{z}=0, \quad \overline{R e}\left(U U_{x}+W U_{z}\right)=-G \sin \theta-P_{x}+U_{z z}, \quad P_{z}=-\delta G \cos \theta,
$$




$$
\bar{u}_{x}+\bar{w}_{z}=0, \quad \bar{m}^{2} \overline{R e}\left(\bar{u}_{\bar{t}}+\bar{u} \bar{u}_{x}+\bar{w} \bar{u}_{z}\right)=-\bar{G} \sin \theta-p_{x}+\bar{u}_{z z}, \quad p_{z}=-\bar{G} \delta \cos \theta .
$$

Here, we have also scaled gravitational effects in the lower layer by setting $G=\bar{G} \delta /(1-\delta) \sim \bar{G} \delta$; these effects enter the upper layer equations at $O(\delta)$, but we have retained all gravitational terms to allow direct comparison with the previous limiting case of section IIB. Note further the presence of inertia in the lower fluid equations at leading order. The boundary and interfacial conditions remain unaltered from Eqs. (2..10), (2..11) and (2..27).

We proceed as in Section 2.2 by integrating the $z$-momentum equations in each layer and making use of the integrated X-momentum equation in the upper layer [expressed by Eq. (2..29) with $U$ given by the closure relation, Eq. (2..33)] and the normal stress boundary condition; this, then, yields the same form for the pressure gradient in the lower layer as in (2..35). We use integral theory in the lower layer in conjunction with the following closure relation for $\bar{u}$, which satisfies the boundary conditions at the interface and bottom wall

$$
\bar{u}=z \tau_{i}+\frac{3}{2 h}\left(\frac{\tau_{i}}{2}-\frac{q}{h^{2}}\right)\left(z^{2}-2 h z\right)
$$

where $\tau_{l}$ is obtained from (33) as before. Integration of the $x$-momentum equation in the lower layer from 0 to $h$, followed by substitution of Eqs. (2..33) and (2..35) into this equation yields an evolution equation for the flow rate in the lower layer, $q$

$$
\bar{m}^{2} \overline{R e}\left(q_{\bar{t}}+I_{x}\right)=-3 \frac{q}{h^{2}}+6 / 5 \overline{R e} \frac{h h_{x}}{(1-h)^{3}}+\frac{3(3+h)}{(1-h)^{3}}-\bar{G} h\left(\sin \theta+\delta \cos \theta h_{x}\right)+\frac{h h_{x x x}}{\overline{C a}},
$$

where

$$
I \equiv \int_{0}^{h} \bar{u}^{2} d z=\frac{3}{10 h}\left(4 q^{2}+\frac{h^{2} q}{(1-h)^{2}}+\frac{h^{4}}{(1-h)^{4}}\right) .
$$

This equation is coupled to

$$
h_{\bar{t}}+q_{x}=0,
$$

which was obtained via integration of the continuity equation in the lower layer and application of the kinematic and wall boundary conditions. Introduction of $(x, \bar{t})=(3 \overline{C a})^{-1 / 3}(\hat{x}, \hat{t})$ finally yields a rescaled evolution equation for $q$ (after suppressing the carets), which is coupled to Eq. (2..41):

$$
q_{t}+I_{x}=\frac{6}{5 \bar{m}^{2}}\left(\frac{h h_{x}}{(1-h)^{3}}+\frac{1}{S}\left[-\frac{q}{h^{2}}+\frac{3+h}{(1-h)^{3}}-\frac{\bar{G}}{3} h\left(\sin \theta+\Delta \cos \theta h_{x}\right)+h h_{x x x}\right]\right),
$$

where the definitions of $S, \bar{G}$ and $\Delta$ remain unaltered from Section 2.2. 


\subsection{Limiting cases}

Here, we examine a number of limiting cases of interest and elucidate the connections between Eqs. (2..36), (2..41) and (2..42) and related equations in the literature used to model the dynamics of single and two-layer flows. We consider Eq. (2..36) first.

First we consider downwards inclined flow with strong gravitational effects and negligible inertia; we set $S \rightarrow 0$ and $(x, t, h)=\left(\frac{3}{\hat{G}}\right)^{1 / 2}(\hat{x}, \hat{t}, \hat{h})$ in which $-\bar{G} \sin \theta=\hat{G}$ so that Eq. (2..36) becomes (after suppressing the carets)

$$
h_{t}+\left(h^{3}\right)_{x}+\left[h^{3} h_{x x x}-\cot (\alpha)\left(3 C a^{1 / 3}\right) h^{3} h_{x}\right]_{x}=0+O\left(\hat{G}^{-1 / 2}\right),
$$

where we have set $\theta=-\alpha$; note that the interfacial stress term in Eq. (2..36), $h^{2}(3+h) /(1-h)^{3}$, is a higher order term. This equation has been derived previously in the literature using lubrication theory to model the dynamics of films flowing down inclined planes in the presence of contact lines [24]. Equation (2..43) admits travelling wave solutions that exhibit thickened ridges in the vicinity of the contact lines; these ridges are unstable to transverse disturbances, which give rise to fingering. Detailed modelling of these fingering patterns has been carried out using the twodimensional version of Eq. (2..43) [25].

In the limit of negligible inertia and weak gravitational effects, we set $S \rightarrow 0, x=3^{-3} \bar{G}^{2} \hat{t}$, $t=\bar{G}^{-1} \hat{t}$ and $h=3^{-4} \bar{G}^{3} \hat{h}$. As a result, Eq. (2..36) reduces to (after suppressing the carets)

$$
\begin{gathered}
h_{t}+\left[h^{2}+h^{3} h_{x x x}\right]_{x}=0+O\left(\bar{G}^{3}\right) \\
h_{t}+q_{x}=0 .
\end{gathered}
$$

In the absence of intermolecular forces and substrate translation, Eq. (44) has previously been used to study the dynamics and stability of thin films climbing up vertical substrates under the action of thermally-induced Marangoni stresses [25].

Equation (2..36) also reduces to an equation studied by Matar et al. [23] by either $\bar{G} \rightarrow 0$ and $\theta \neq 0$, or $\bar{G} \neq 0, \theta=0$ and $C a \rightarrow 0$; the latter limit corresponds to that of horizontal channels and very strong capillary effects. Note that Matar et al. [23] have also shown that it is possible to derive a Benney-type equation and the Kuramoto-Sivashinsky equation from Eq. (2..36).

Equations (2..41) and (2..42), which were derived in the limit of small density ratios, reduce to Eq. (2..36) by letting $\bar{m} \rightarrow 0$. It is also possible to set $\theta=-\pi / 2, x=\bar{G}^{-5 / 11} \bar{x}, t=\bar{G}^{-8 / 11} \bar{t}$, $h=\bar{G}^{-4 / 11} \bar{h}$ and $q=\bar{G}^{-1 / 11} \bar{q}$ so that Eqs. (2..41) and (2..42) become (after suppressing the caret decoration)

$$
q_{t}+\frac{6}{5}\left(\frac{q^{2}}{h}\right)_{x}=\frac{1}{C}\left[\frac{h}{3}+h h_{x x x}-\frac{q}{h^{2}}\right]+O\left(\bar{G}^{-7 / 11}\right),
$$

where $C \equiv \frac{6}{5 \bar{m}^{2} S}$ is equivalent to the parameter $\delta$ in the Shkadov equations [26,27] to which Eqs. (2..41) and (2..42) reduce for $\theta=-\pi / 2$ and $\bar{G} \gg 1$. These equations have been previously used to study the dynamics of falling films. Note that it is also possible to derive the Kuramoto-Sivashinsky equation from Eq. (2..45) for travelling waves of sufficiently small amplitude. 


\section{Results}

\subsection{Linear stability analysis}

We linearise Eq. (2..36) about a steady-state thickness, $h_{0}$, using normal modes: $h(x, t)=h_{0}+$ $h_{1} e^{i k x} e^{\omega t}$; here, $k$ and $\omega$ represent a real disturbance wavenumber and a complex growth rate, respectively, and $h_{1} / h_{0} \ll 1$. This leads to the following expression for $\omega$ :

$$
\omega=k^{2} h_{0}^{3}\left[\left(\frac{S}{\left(1-h_{0}\right)^{3}}-\frac{\bar{G}}{3} \Delta \cos \theta\right)-k^{2}\right]+i \frac{k h_{0}\left[\bar{G} \sin \theta\left(1-h_{0}\right)^{4} h_{0}-6\left(1+h_{0}\right)\right]}{\left(1-h_{0}\right)^{4}} .
$$

Inspection of Eq. (3..1) reveals that the flow is linearly unstable provided that

$$
S>S_{c} \equiv \frac{\bar{G}}{3} \Delta \cos \theta\left(1-h_{0}\right)^{3} .
$$

Then wavenumbers in the range $0<k<k_{c}$ are unstable, where $k_{c}$ is the wavenumber associated with the cut-off mode, given by

$$
k_{c}=\left(\frac{S}{\left(1-h_{0}\right)^{3}}-\frac{\bar{G}}{3} \Delta \cos \theta\right)^{1 / 2}
$$

A most dangerous mode also exists with wavenumber $k_{m}=k_{c} / \sqrt{2}$ and growth rate $\omega_{m}=k_{c}^{4} h_{0}^{3} / 4$. From Eq. (3..2), it is clearly seen that although inertia is destabilising for all values of $\theta$, with its effect becoming more pronounced for increasing $h_{0}$, while the direct effect of gravity is stabilising for $n<1$. However, for a given flow rate $q_{0}$ of the lower fluid, the equilibrium interface height is determined by

$$
q_{0}=\frac{h_{0}^{2}\left(3+h_{0}\right)}{\left(1-h_{0}\right)^{3}}-\frac{\bar{G}}{3} h_{0}^{3} \sin \theta
$$

so that increasing gravity will generally act to increase (decrease) $h_{0}$ for upwards (downwards) inclined flows, as shown in Figure 1a. Hence the overall effect of gravity can be destabilising for fixed flow rates in upwards inclined flows.

The dependence of $S_{c}$ on $h_{0}$ is shown in Figure $1 \mathrm{~b}$ for situations in which $\bar{G}>0$. As shown in Figure 1, decreasing $h_{0}$ and $\bar{G}$ increases $S_{c}$ and is therefore stabilising; this can be interpreted as an increase in velocity $V$ required to drive instability in the two-layer system. Increasing $\Delta$ and/or $\theta$ has the same effect as decreasing $h_{0}$ and $\bar{G}$.

We have also investigated the linear stability characteristics of Eqs. (2..41) and (2..42) by linearising these equations about the following steady state, $h=h_{0}$ and

$$
q=q_{0}=\frac{h_{0}^{2}\left(3\left(3+h_{0}\right)-\bar{G}\left(1-h_{0}\right)^{3} h_{0} \sin \theta\right)}{3\left(1-h_{0}\right)^{3}},
$$

using a normal modes expansion $(h, q)(x, t)=\left(h_{0}, q_{0}\right)+\left(h_{1}, q_{1}\right) e^{(i k x)} e^{\omega t}$ where $k$ and $\omega$ are defined as above. Solution of the linearised equations for the disturbance growth, $\omega_{r}$, leads to the 
(a)
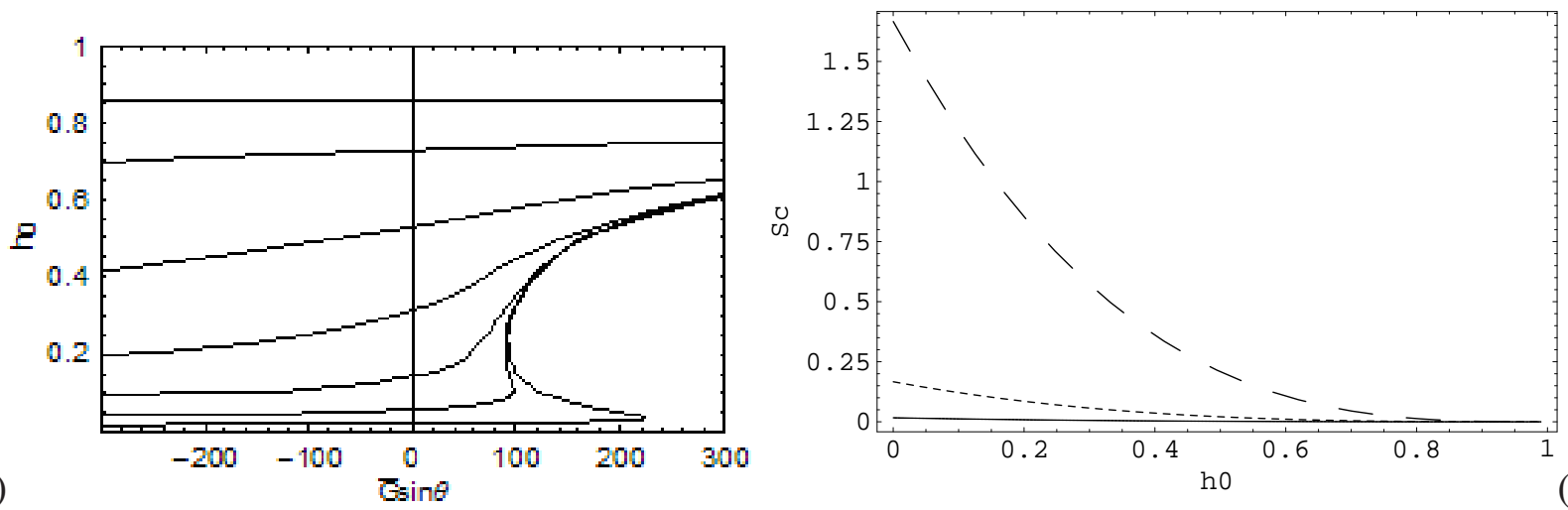

Figure 1: 1 (a) The dependence of $h_{0}$ on $\bar{G} \sin \theta$ for given flow rates $q_{0}=$ $10^{-3}, 10^{-2}, 10^{-1}, 1,10,10^{2}, 10^{3}$ (upper curve). Note the existence of three solutions for large $\bar{G} \sin \theta$ and small $q_{0}$. (b) The dependence of $S_{c}$ on $h_{0}$ at criticality for $\bar{G}=1$ (solid line), $\bar{G}=10$ (dotted line) and $\bar{G}=100$ (dashed line), $\Delta=0.1$ and $|\theta|=\pi / 3$.

results shown in Figure 2. The dispersion curves shown in this figure are paraboloidal with a band of wavenumbers for which $\omega_{r}>0$ indicating the presence of a linear instability. This band exhibits well-defined 'cut-off' and 'most dangerous' modes at intermediate values of $k$, defined as $k_{c}$ and $k_{m}$, respectively; for $k=k_{m}, \omega \equiv \omega_{m}$. As shown in Figure 2, $\omega_{m}$ is maximised for an intermediate value of $\bar{m}$. The value of $k_{c}$, however, increases monotonically with increasing $\bar{m}$. From Figure $2 \mathrm{~b}$, it can be seen that, in the linear regime, declining the channel is destabilising (for fixed $h_{0}$ ), leading to a marginal increase in $k_{m}$ and $\omega_{m}$ but a more significant increase in $k_{c}$. The effect of increasing $\Delta$ was found to be strongly stabilising due to a concomitant increase in the relative significance of the stabilising hydrostatic component of the gravitational forces, as shown in Figure 2c. Finally, increasing $\bar{G}$ (with fixed $h_{0}$ ), destabilises linear disturbances in negatively inclined channels, as can be seen upon inspection of Figure 2d,e. The linear theory predictions will be compared with the results of the numerical simulations of the flow behaviour in the nonlinear regime. These are presented next.

\subsection{Numerical simulations}

The numerical procedure used to carry out the computations in this work is based on finite element collocation to discretise the spatial derivatives and Gear's method to advance the solution in time. Up to 8000 grid points were used to obtain numerical solutions in the range $0<x<L_{c}=300$ starting from

$$
\begin{gathered}
h(x, 0)=h_{0}+0.01 \times e^{-10(x-50)^{2}}, \\
q(x, 0)=q_{0}=\frac{h_{0}^{2}\left(3\left(3+h_{0}\right)-\bar{G}\left(1-h_{0}\right)^{3} h_{0} \sin \theta\right)}{3\left(1-h_{0}\right)^{3}}
\end{gathered}
$$



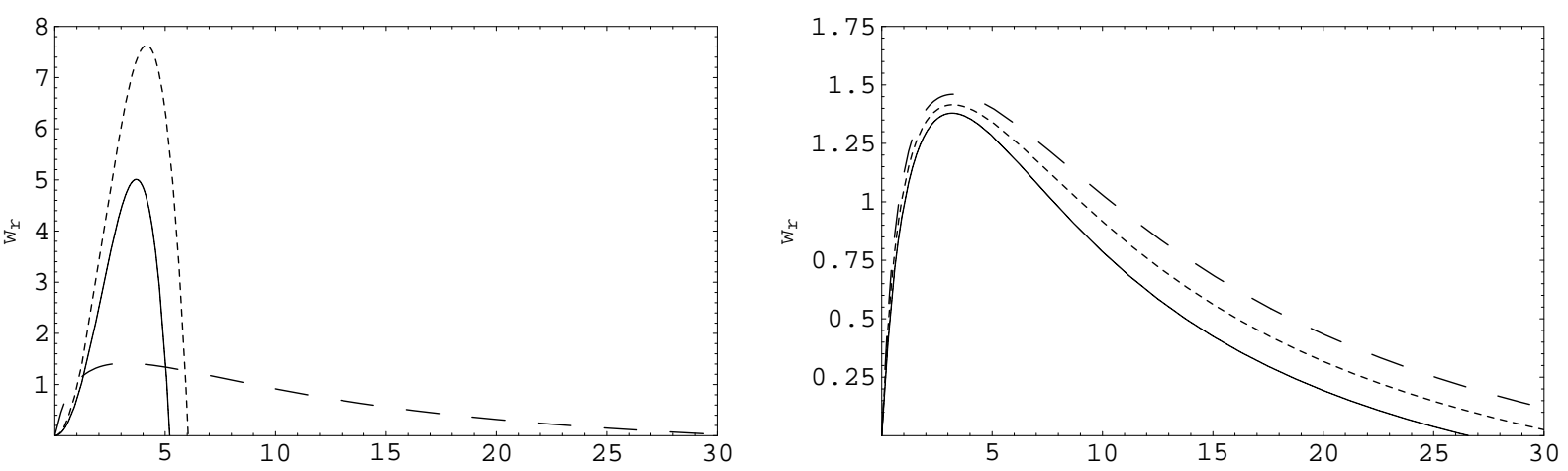

(a)
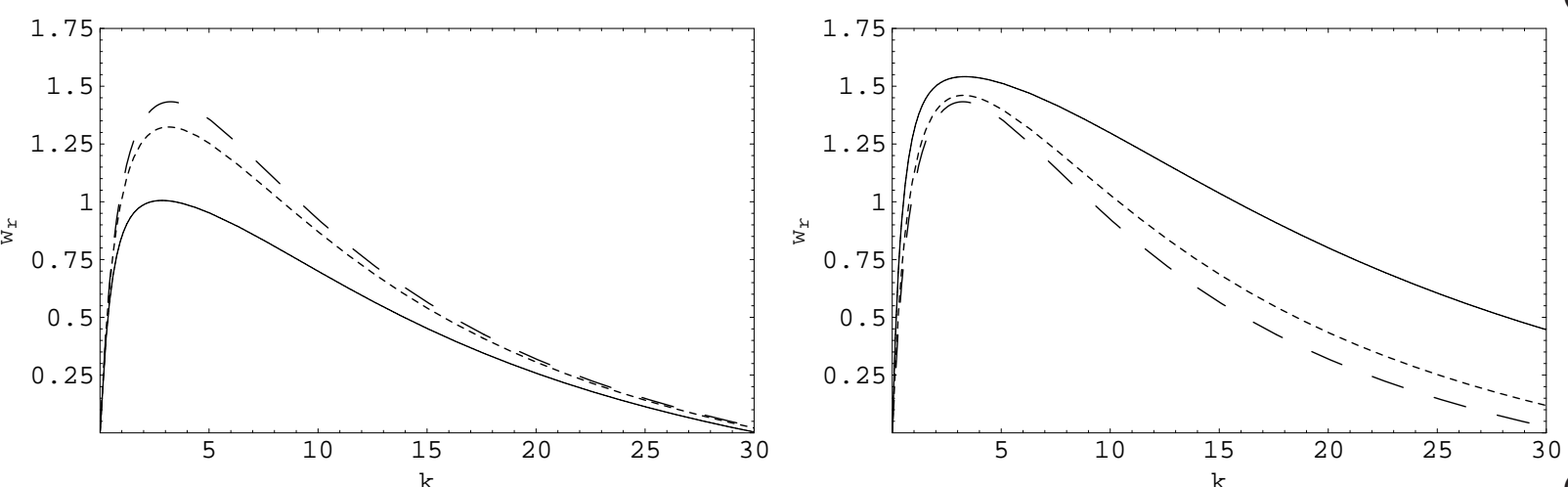

(b)

(c)

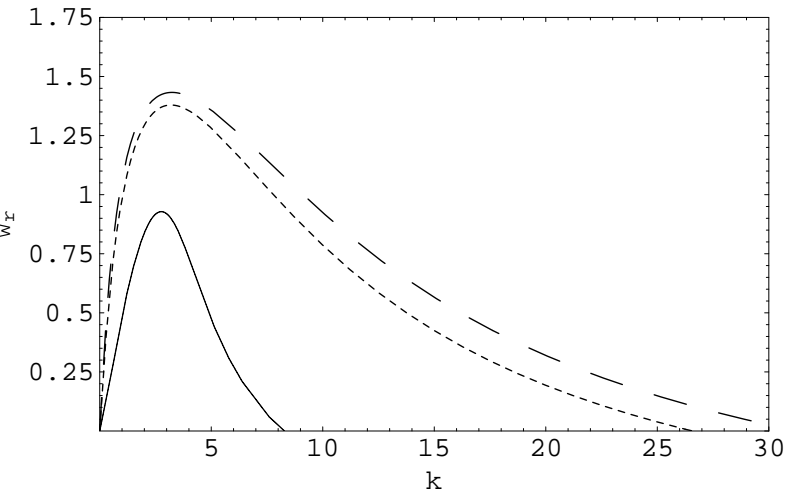

(d)

(e)

Figure 2: The dependence of the growth rate on system parameters. (a) Effect of $\bar{m}: \bar{m}=$ 1, 0.1, 0.01 shown by dashed, dotted and solid lines, respectively; (b) effect of $\theta: \theta=-\pi / 3,0, \pi / 3$ shown by dashed, dotted and solid lines, respectively; (c) effect of $\Delta: \Delta=0,2,10$ shown by dashed, dotted and solid lines, respectively; effect of $\bar{G}: \bar{G}=0,20,100$ shown by dashed, dotted and solid lines, respectively, with $\theta=-\pi / 3$ and $\theta=\pi / 3$ in (d) and (e), respectively. Unless stated otherwise, the parameter values are $\bar{m}=1, h_{0}=0.3, S=10, \bar{G}=20, \theta=0$ and $\Delta=0.3$. 
subject to the following boundary conditions,

$$
(h, q)(0, t)=\left(h_{0}, q_{0}\right), \quad h_{x x x}(0, t)=0, \quad\left(h_{x}, h_{x x x} q_{x}\right)\left(L_{c}, t\right)=0 ;
$$

here, $L_{c}$ is the length of the computational domain. The initial condition for $q$, given by Eq. (3..5), and associated boundary conditions are imposed only when Eqs. (2..42) and (2..43) are solved. In order to characterise the interfacial waviness, and provide a measure of instability, the following integral function is introduced

$$
W(t)=\frac{1}{L_{c}} \int_{0}^{L_{c}}\left(h-h_{0}\right)^{2} d x .
$$

We begin the presentation of our results by examining the spatio-temporal evolution of the interface associated with the first distinguished limit, $m \sim \delta$ and $n=O(1)$, for a horizontal channel; this is shown in Figure 3 for $h_{0}=0.3, S=10, \bar{G}=10, \theta=0$ and $\Delta=0.3$. The perturbation provided by the initial condition is convected downstream and leads to the formation of large-amplitude waves, as shown by the space-time plot in Figure 3a. Two regions may be distinguished and are shown in Figure 3b: a region populated by closely-spaced waves and a mean thickness lower than $h_{0}$, and another region located downstream of the first one, which exhibits solitary-wave formation (see Figure 3c); these correspond to waves of large amplitude separated by regions in which the interface is flat and is approximately equal to $h_{0}$. Those results are similar to these obtained by Matar et al. [23] who also showed that increasing $h_{0}$ and/or $S$ leads to interface destabilisation characterised by the formation of waves of larger amplitude and the possibility of channel bridging. We focus below on the effect of gravity on the dynamics.

The effect of $\bar{G}$ on the $h$-profiles is shown in Figure 4 for the case of horizontal channels. It is clearly seen that increasing $\bar{G}$ stabilises the interface; this is reflected by a decrease in wave amplitude and interfacial waviness, as shown in Figure $4 \mathrm{~d}$. This stabilising effect is due to the hydrostatic component of the gravitational forces, which gives rise to interfacial flattening; this is in agreement with the linear theory predictions [see Eq. (2..45)] which indicate that increasing the significance of the hydrostatic component increases the critical value of $S$ for the onset of wave formation. Similar trends are observed upon increasing $\Delta$ (not shown), as expected.

We have also studied the effect of channel inclination on the dynamics. As shown in Figure 5, positive channel inclinations destabilise the flow giving rise to an increase in interfacial waviness (see Figure 5d). This is due to an associated increase in the magnitude of the interfacial pressure gradient, which is given by

$$
\left(\left.p\right|_{h}\right)_{x}=-G\left(\sin \theta+\delta h_{x} \cos \theta\right)-\frac{6}{5(1-h)^{3}}\left(1-+\overline{R e} h_{x}\right)-\frac{h_{x x x}}{\bar{C} a}
$$

that drives the formation of waves of larger amplitude than in the $\theta=0$ and $\theta<0$ cases.

Next, we turn our attention to the dynamics associated with the second distinguished limit, $m \sim \delta^{1 / 2}$ and $n \sim \delta$. We first investigate the effect of increasing $\bar{m}$ on the dynamics in horizontal channels. As mentioned in Section 2.4 , we would expect Eqs. (2..41) and (2..42) to reduce to Eq. (2..36) in the limit of small $\bar{m}$. As shown in Figure 5, a decrease in $\bar{m}$ leads to a transition in wave structure: at relatively large values of $\bar{m}$, the typical large-amplitude pulses are absent; 
(a)

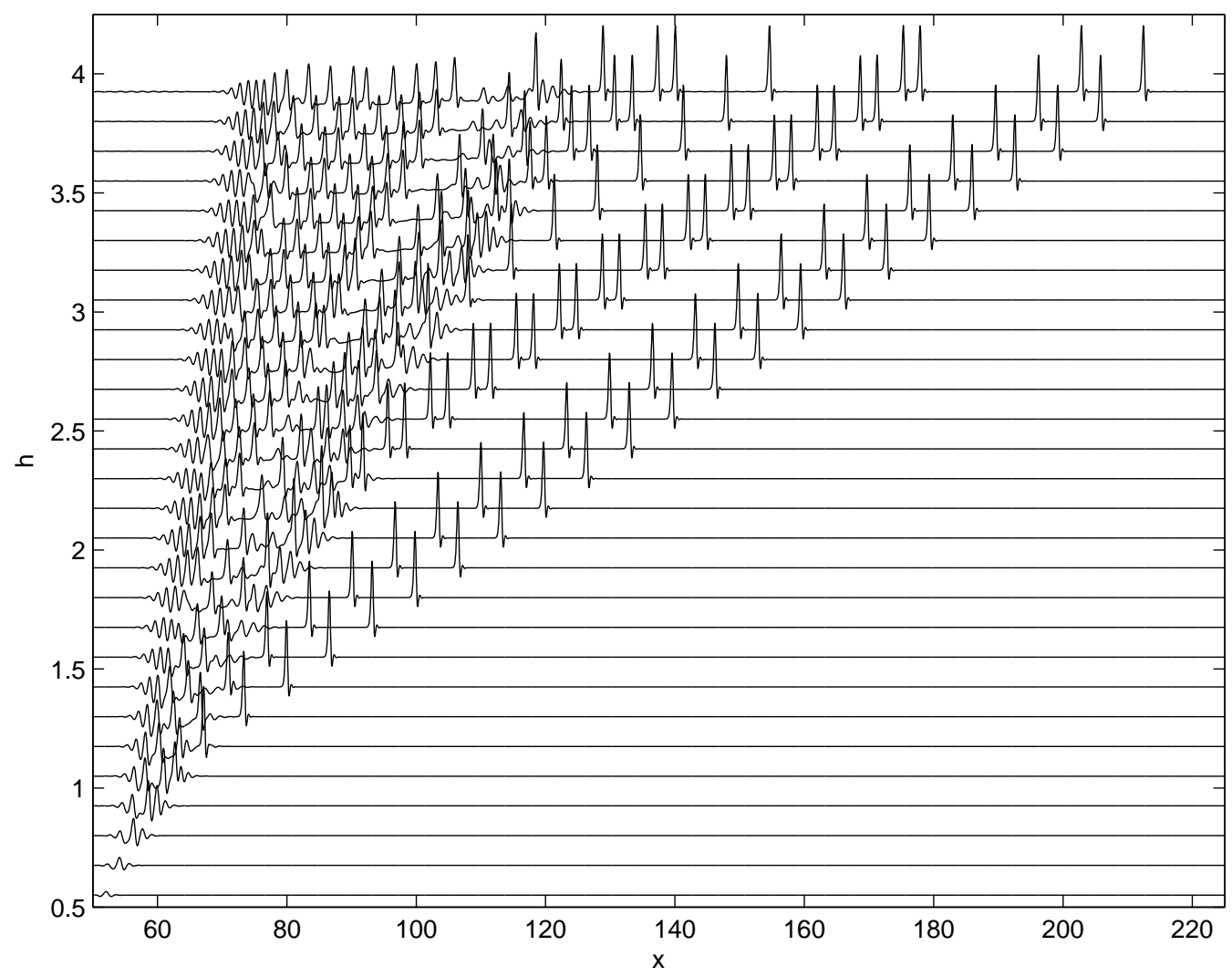

(b)

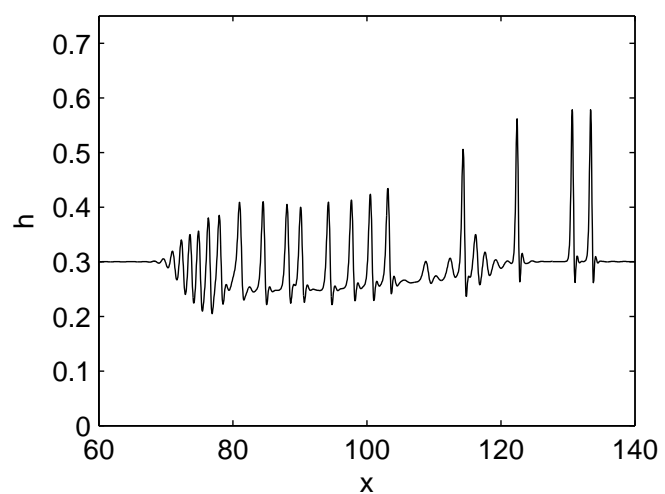

(c)

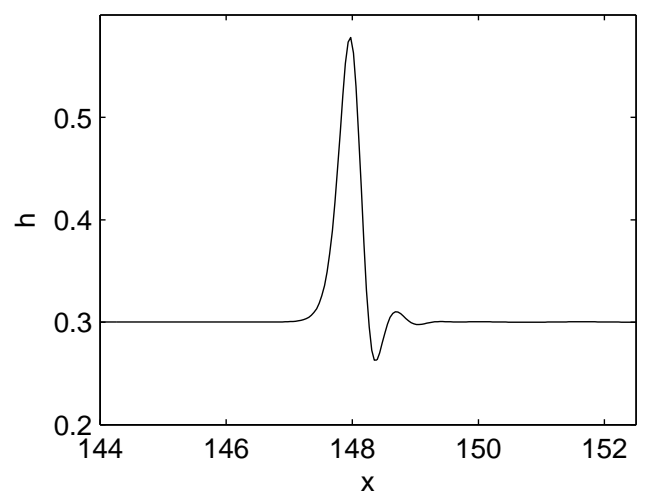

Figure 3: Space-time plot showing the spatio-temporal evolution of the interface, (a), generated with $h_{0}=0.3, S=10, \bar{G}=10, \theta=0$ and $\Delta=0.3$, for $t=0.2-5.6$ and 28 equal time steps. Panels (b) and (c) show enlarged views of the regions $60 \leq x \leq 140$ and $144 \leq x \leq 152.5$, respectively, for $t=5.4$ and the same parameters are those used to generate the results shown in (a). 
(a)

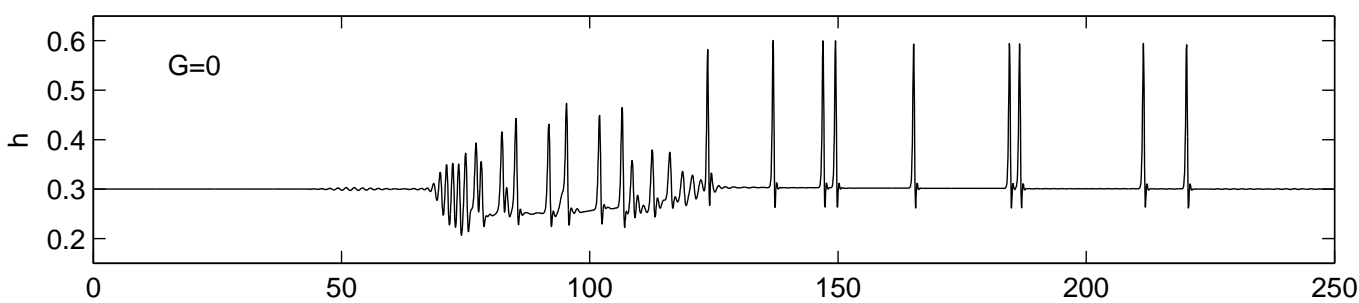

(b)

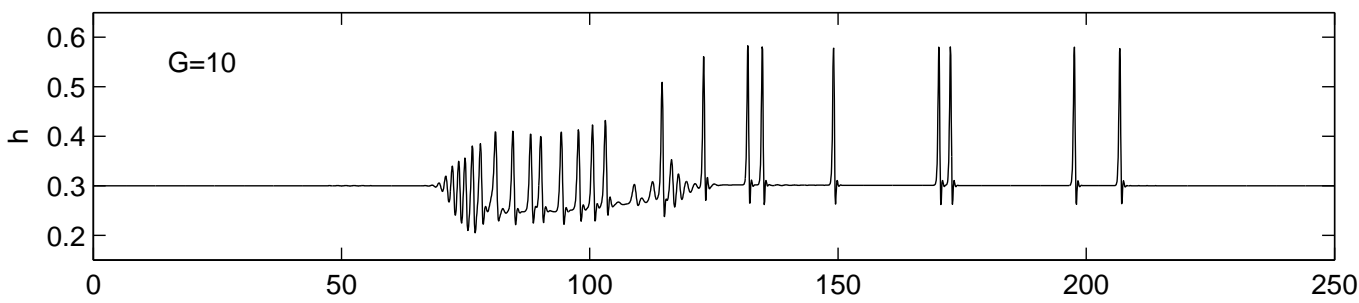

(c)
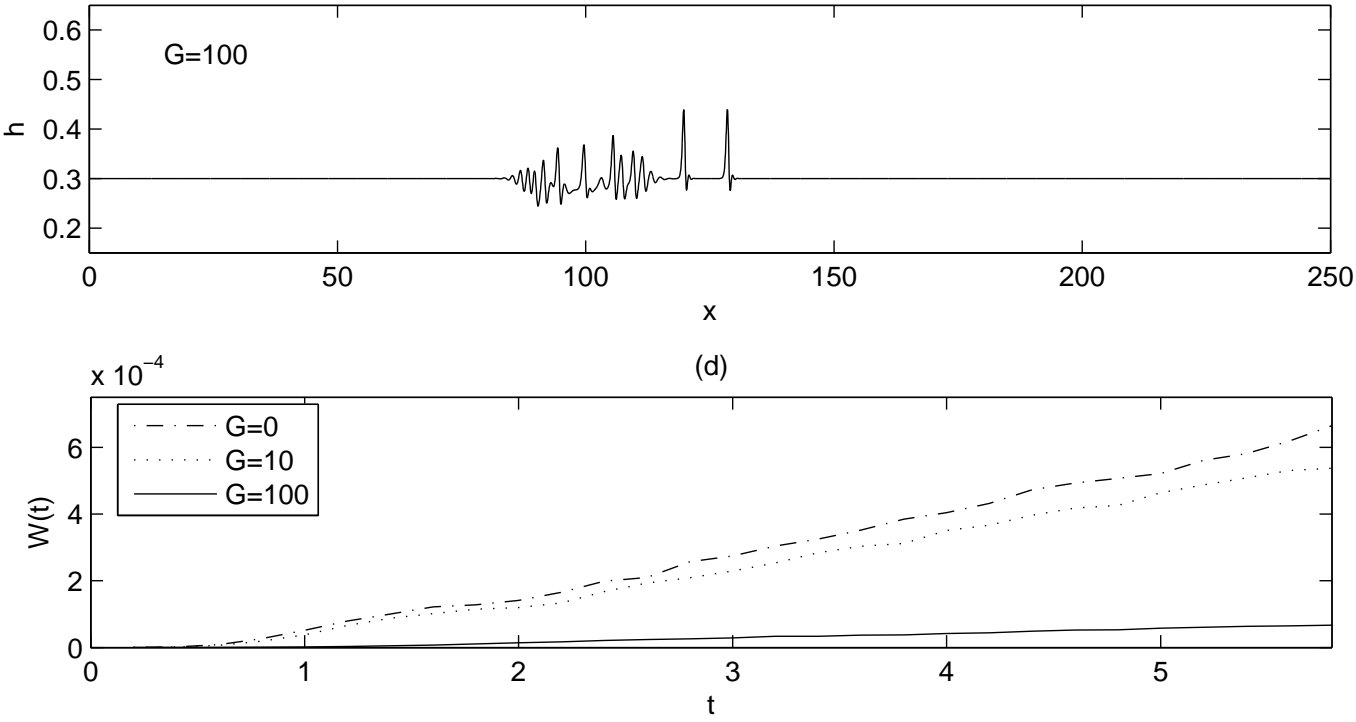

Figure 4: The effect of $\bar{G}$ on the interfacial profiles, shown in (a)-(c) at $t=5.6$, and the interfacial waviness, shown in (d). The rest of the parameter values are unchanged from Figure 3. 
(a)

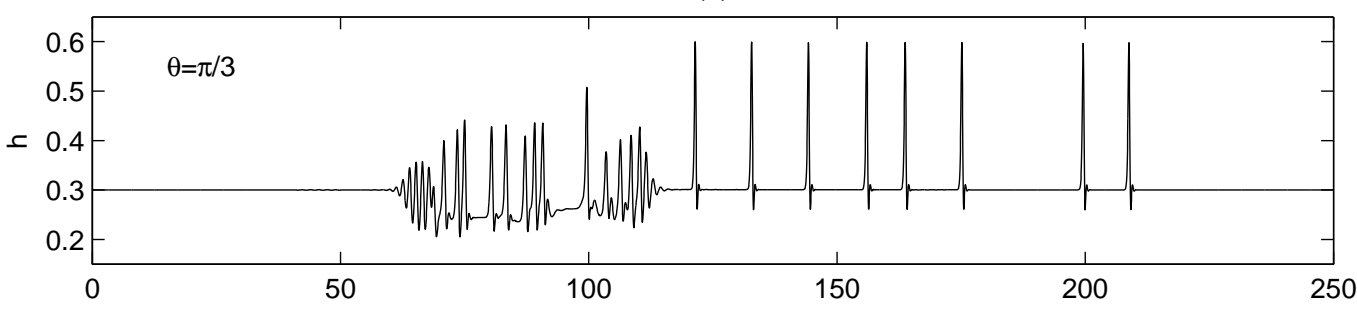

(b)

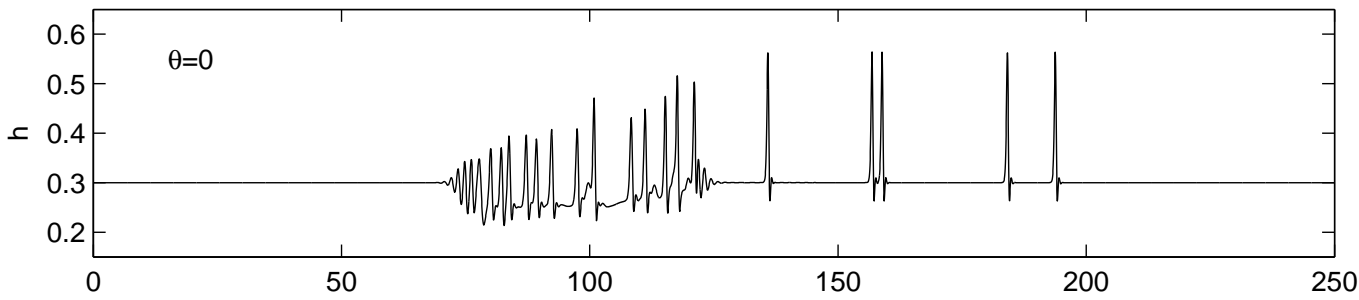

(c)
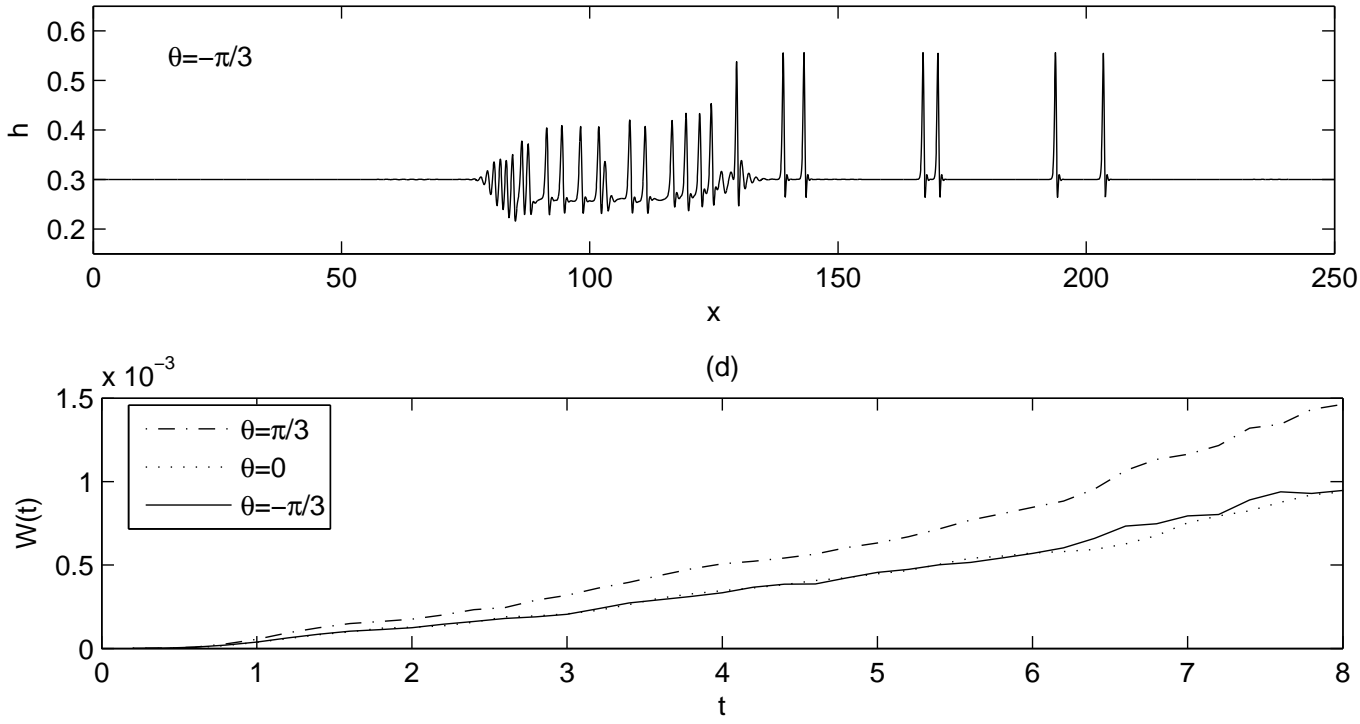

Figure 5: The effect of $\theta$ on the interfacial profiles, shown in (a)-(c) at $t=5.6$, and the interfacial waviness, shown in (d). Here, $\bar{G}=20$ and the rest of the parameter values are unchanged from Figure 3 . 
(a)

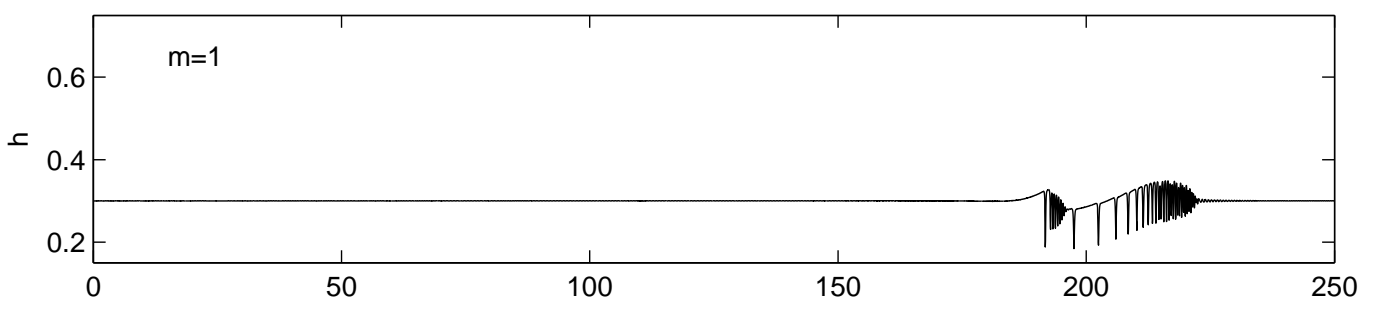

(b)

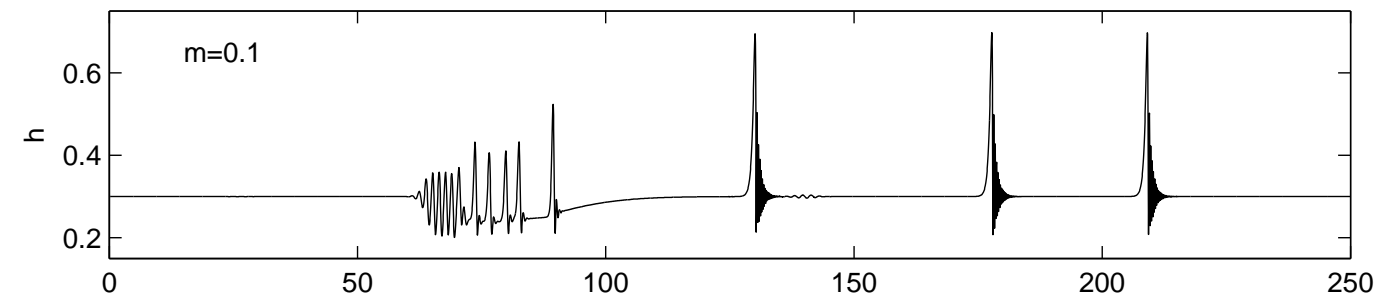

(c)

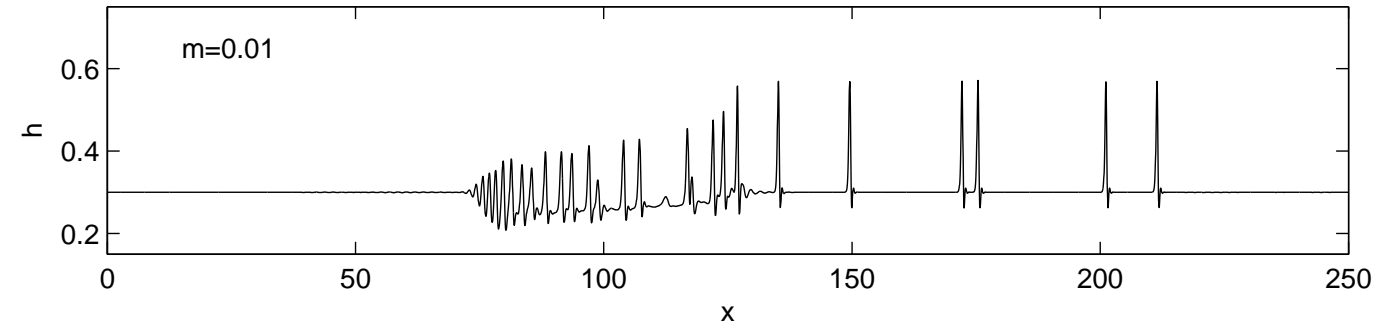

(d)

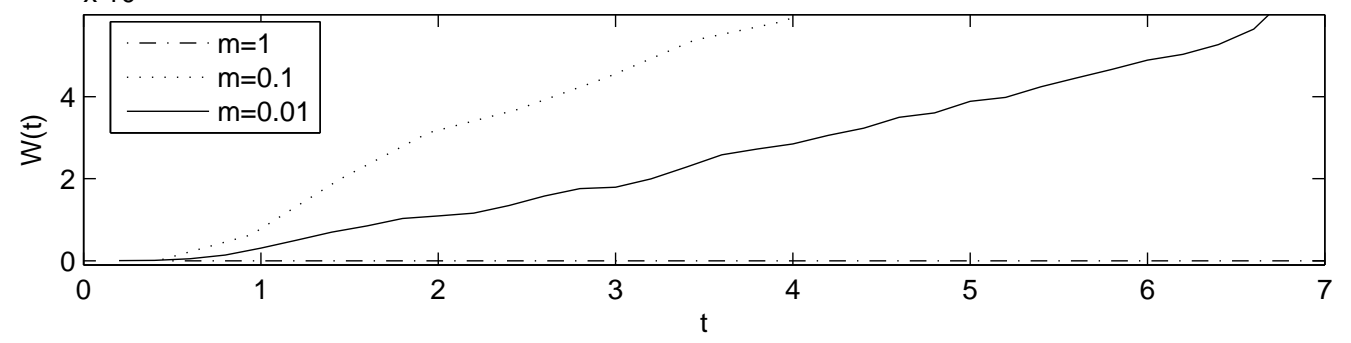

Figure 6: The effect of $\bar{m}$ on the interfacial profiles, shown in (a)-(c) at $t=19.5,3.95,6.4$, respectively, and the interfacial waviness, shown in (d). The rest of the parameter values are $h_{0}=0.3, S=10, \bar{G}=20$, and $\Delta=0.3$. 
(a)

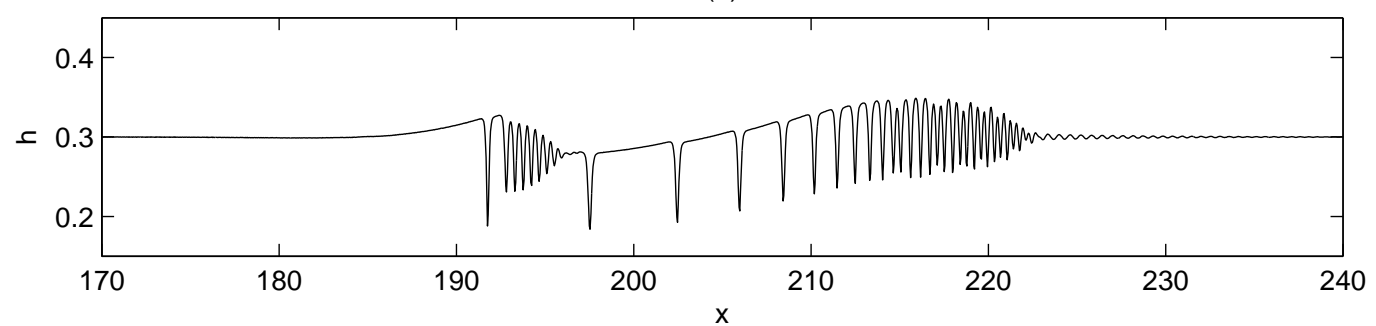

(b)

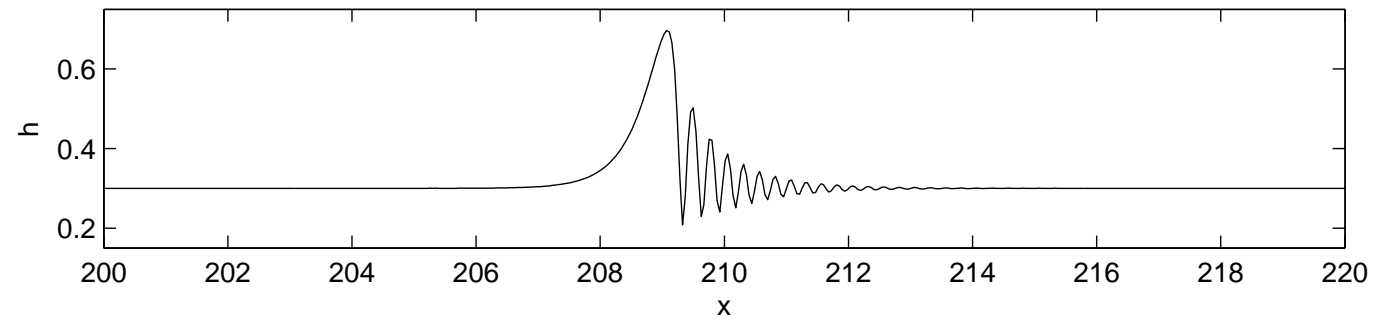

(c)

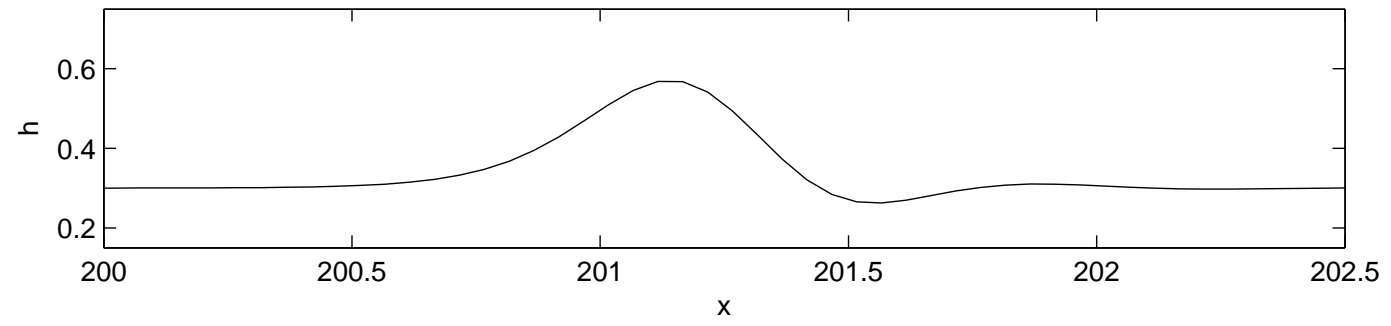

Figure 7: Enlarged views of the regions $170<x<240,200<x<220$ and $200<x<202.5$ in panels (a)-(c) of Figure 7, respectively. 
(a)

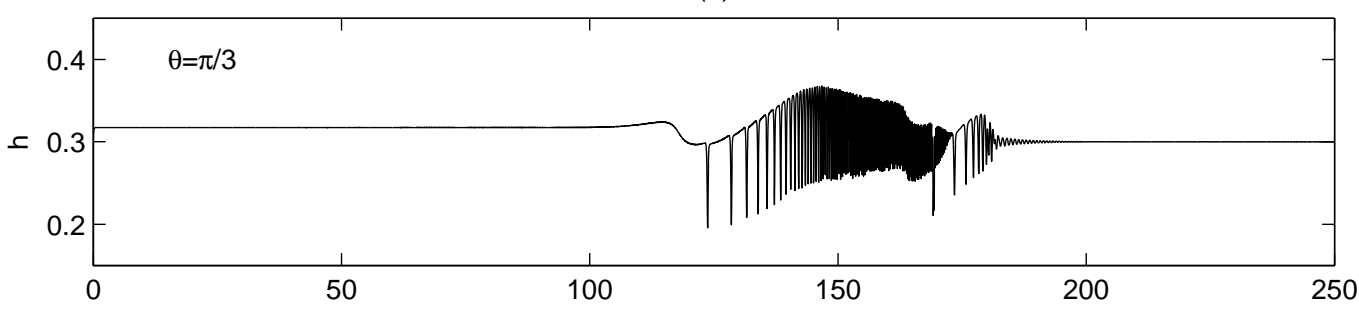

(b)

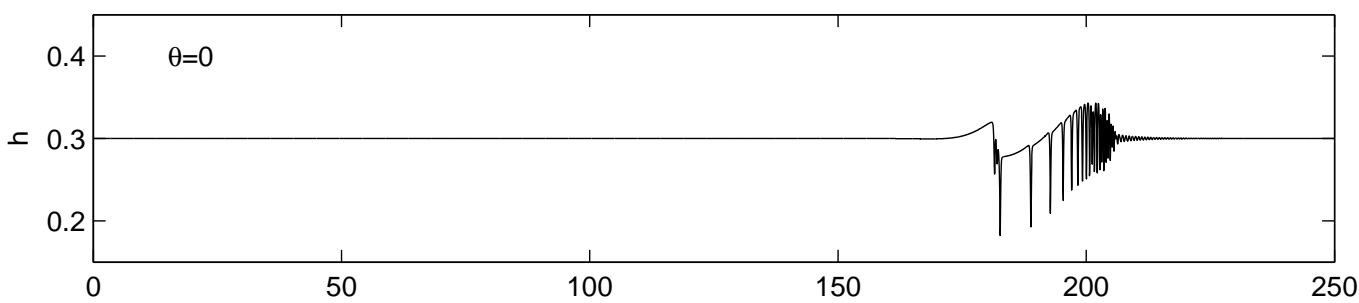

(c)
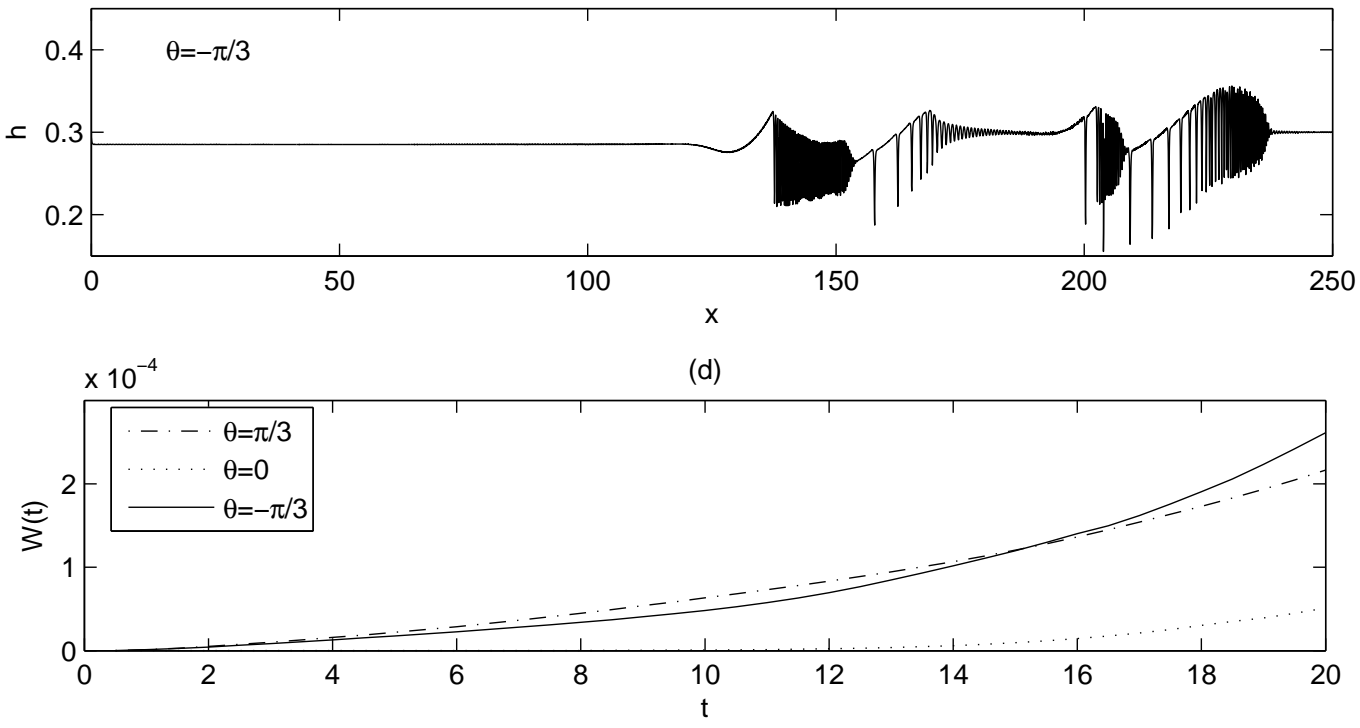

Figure 8: The effect of $\theta$ on the interfacial profiles, shown in (a)-(c) at $t=18$, and the interfacial waviness, shown in (d). The rest of the parameter values are $\bar{m}=1, h_{0}=0.3, S=10, \bar{G}=20$ and $\Delta=0.3$. 
instead, the waves appear to travel as packets which do not radiate travelling waves (see also Figure 7 a for an enlarged view of these waves). With decreasing $\bar{m}$, the interfacial dynamics resemble those associated with the first distinguished limit, shown in Figure 4 and Figure 5. The degree of instability appears to be maximal for an intermediate value of $\bar{m}$ : as shown in Figure $6 \mathrm{~d}, W(t)$ for $\bar{m}=0.1$ exceeds that associated with $\bar{m}=1$ and $\bar{m}=0.01$. The solitary waves at $\bar{m}=0.1$ also have larger amplitude than those at $\bar{m}=0.01$ and are preceded by more pronounced capillary ripples, the so-called "bow" waves, as shown in Figure 7b,c; these solitary waves resemble those observed in falling film dynamics. These results, obtained in the nonlinear regime, are in agreement with the predictions of linear theory, discussed in Section 3.1 (see Figure 2a), which also indicate that the growth rate is maximised for an intermediate value of the viscosity ratio.

(a)
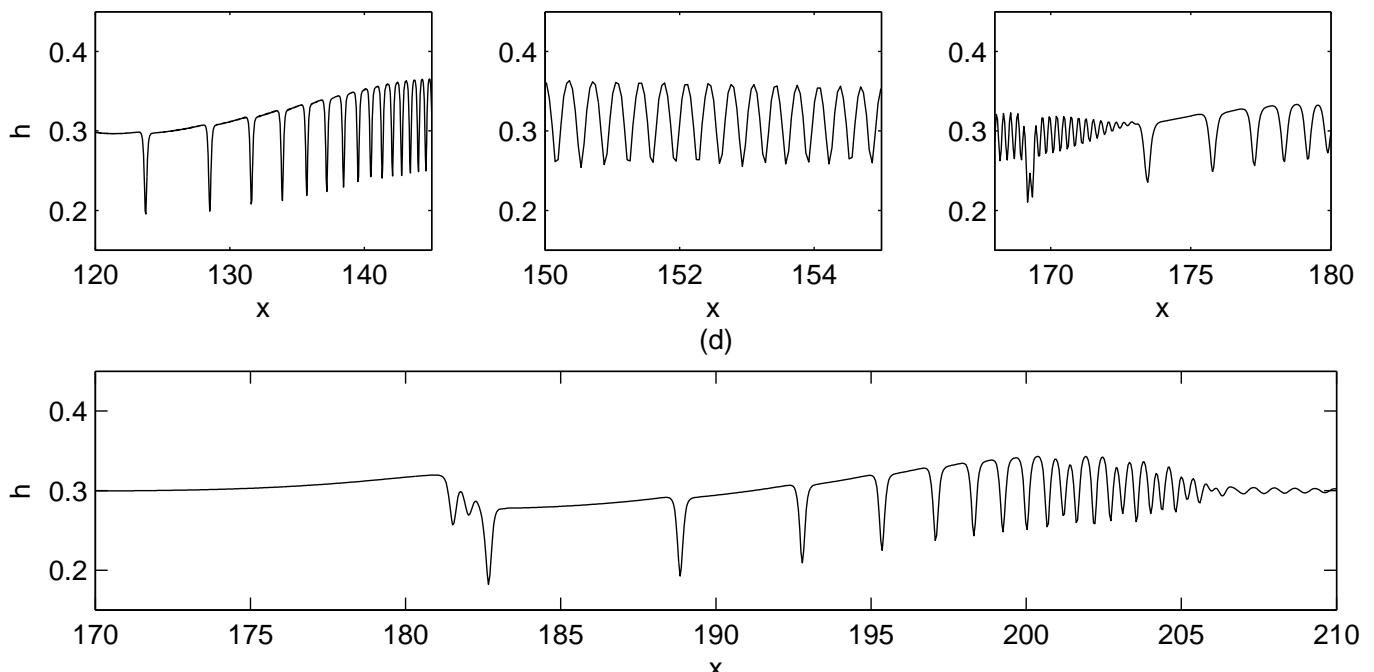

(e)

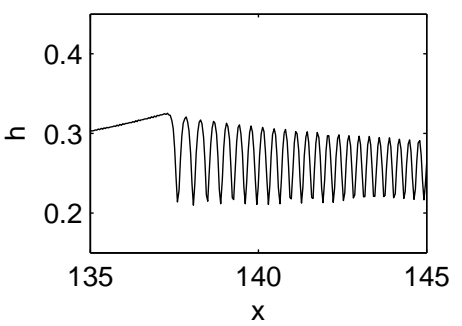

(b)

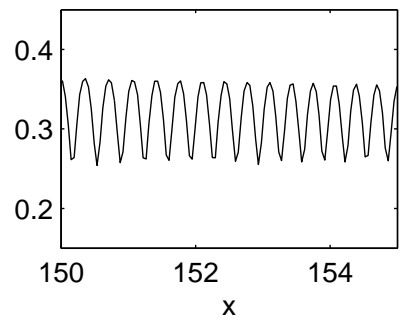

(c)

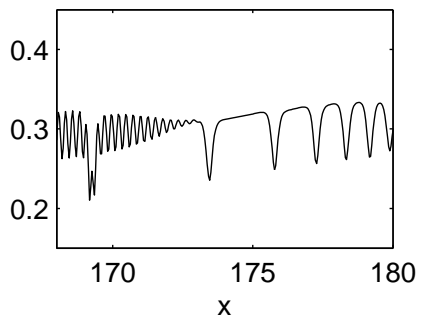

(f)

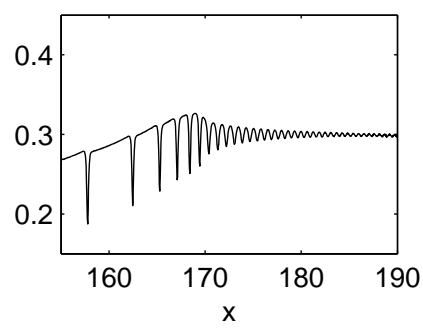

(g)

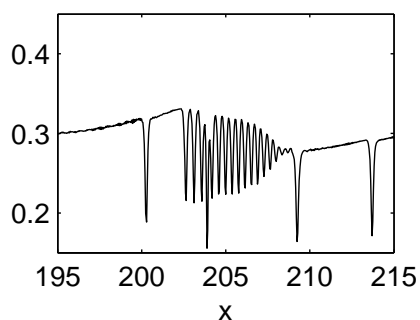

Figure 9: Enlarged views of various regions in panels (a)-(c) of Figure 8 shown in (a)-(c), (d) and (e)-(g), respectively.

The close up view in Figure 7a shows that for this relatively large value of $\bar{m}$, there are in fact pulse like wave structures, but these are "upside down"; pulses of the upper fluid penetrate the lower fluid. This is what we would normally expect if the upper fluid were more viscous. In this case, the lower fluid is much more viscous, but the interaction is apparently controlled by the scaled viscosity ratio $\bar{m}$.

We have also studied the effect of channel inclination on the dynamics in the second distin- 

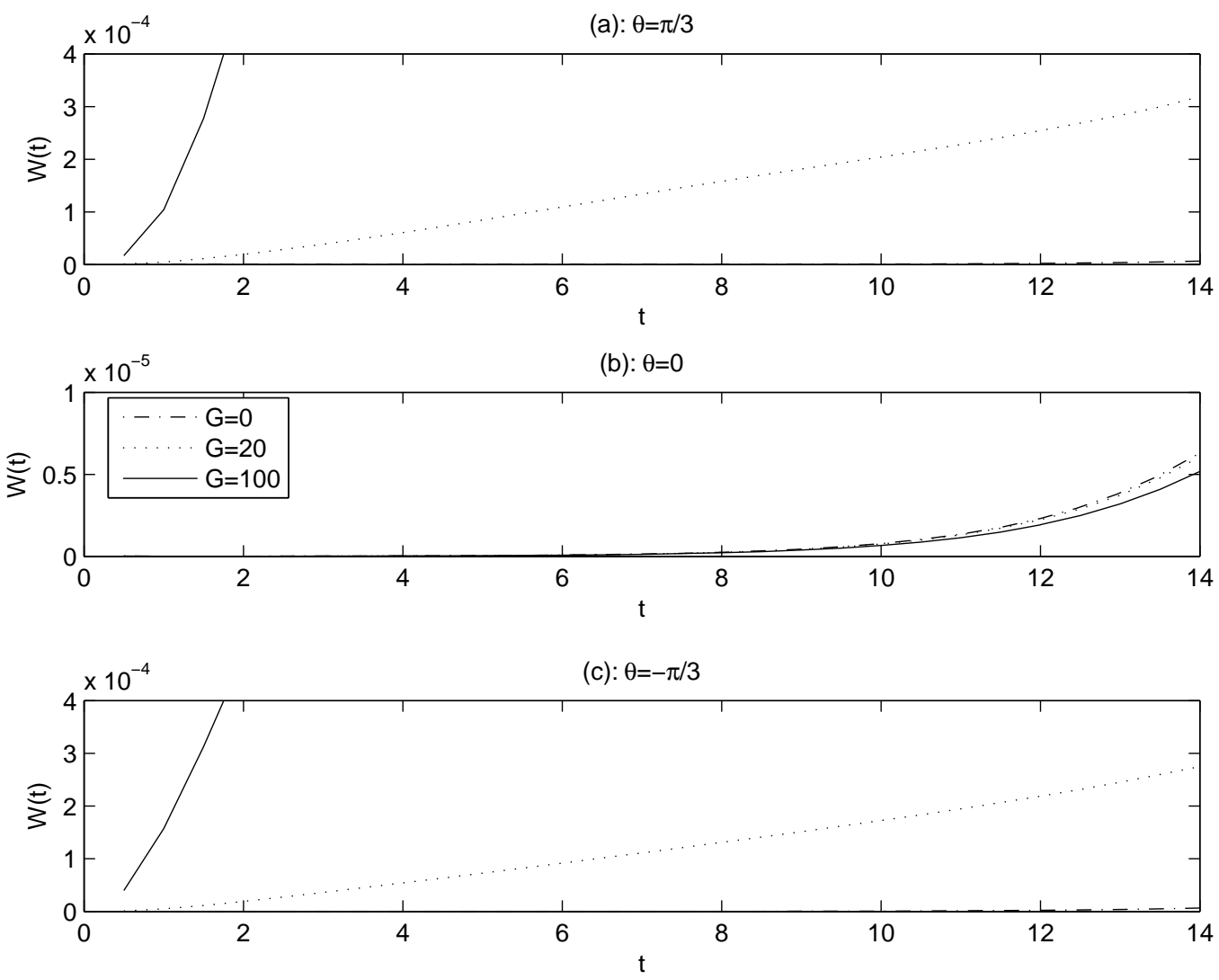

Figure 10: The effect of increasing $\bar{G}$ on the interfacial waviness for $\theta=\pi / 3,0,-\pi / 3$. The rest of the parameters remain unchanged from Figure 8. 
guished limit. In Figure 8, it is seen that variation of the channel inclination does not lead to a qualitative change in the overall structure of the waves; these appear to retain their integrity as travelling wave packets that do not radiate solitary waves. As shown in Figure 8 and Figure 9, these packets straddle two flat interfacial regions: an elevated 'lip' marks the transition from the flat region on the inlet side, while the transition to the other flat region downstream is marked by interfacial oscillations of relatively high frequency. The packet interior is populated by waves whose wavelength decreases with increasing distance downstream.

Inclining the channel appears to destabilise the interface, as illustrated in Figure 8d, which shows that $W(t)$ for $|\theta|=\pi / 3$ is larger than that associated with the horizontal channel case. The results shown in Figure 8d also indicate that negative inclinations are slightly more destabilising than positive ones, in agreement with the predictions of the linear stability analysis. For $\theta=-\pi / 3$, the interfacial evolution is accompanied by the formation of two wave packets, which are separated by a flat region, as shown in Figure $8 \mathrm{c}$ and Figure $9 \mathrm{e}-\mathrm{g}$.

The effect of varying $\bar{G}$ on the interfacial waviness has also been studied for the case of horizontal and inclined channels. As shown in Figure 10, increasing $\bar{G}$ is destabilising for $|\theta|>0$; this effect is marginally more pronounced for positively inclined channels.

\section{Concluding remarks}

We have considered the pressure-driven flow of two immiscible layers in inclined channels. Evolution equations that describe the spatio-temporal evolution of the interface were derived using asymptotic reduction, boundary-layer theory and the Karman-Polhausen approximation for the case of high viscosity contrasts. In the case of density ratios of order unity, the dynamics are modelled using a single evolution equation for the interfacial position, while for high density contrasts, an additional equation must be solved for the flow rate in the more viscous layer. Our results indicate that the dynamics associated with high viscosity contrasts and order unity density ratios is characterised by the formation of large-amplitude solitary wave formation, which becomes more pronounced for positive channel inclinations. The interfacial evolution for the case of high viscosity and density contrasts is characterised by the formation of inverted wave packets, which do not radiate solitary waves. Future work will focus on comparisons of our modelling predictions against experimental data.

\section{References}

[1] G.F. Hewitt. Handbook of Multiphase Systems, ed. G. Hestroni, Hemisphere, New York, Chap. 2, 1982.

[2] P.Y. Lin, T.J Hanratty. Prediction of the initiation of slugs with linear-stability theory. Int. J. Multiphase Flow, 12 (1986), 79-98.

[3] N. Andritsos, T.J. Hanratty. Interfacial instabilities for horizontal gas-liquid flows in pipelines. Int. J. Multiphase Flow, 13 (1987), 583-603. 
[4] Y. Taitel, A.E. Dukler. Model for predicting flow regime transitions in horizontal and nearhorizontal gas-liquid flow. AIChE J., 22 (1976), 47-55.

[5] C.S. Yih. Instability due to viscosity stratification. J. Fluid Mech., 27 (1967), 337-352.

[6] E.J. Hinch. A note on the mechanism of the instability at the interface between two shearing fluids. J. Fluid Mech., 144 (1984), 463-465.

[7] S.G. Yiantsos, B.G. Higgins. Linear stability of plane Poiseuille flow of two superposed fluids. Phys. Fluids, 31 (1988), 3225-3238.

[8] R.H.M. Miesen, B.J. Boersma. Hydrodynamic stability of a sheared liquid film. J. Fluid Mech., 301 (1995), 175-202.

[9] W.C. Kuru, M. Sangalli, D.D. Uphold, M.J. McReady. Linear stability of stratified channel flow. Int. J. Multiphase Flow, 21 (1995), 733-753.

[10] T. Boeck, S. Zaleski. Viscous versus inviscid instability of two-phase mixing layers with continuous velocity profile. Phys. Fluids. 17 (2005), 032106, 1-11.

[11] R.I. Issa, M.H.W. Kemp. Simulation of slug flow in horizontal and nearly horizontal pipes with the two-fluid model. Int. J. Multiphase Flow, 29 (2003), 69-95.

[12] M. Bonizzi, R.I. Issa.On the simulation of three-phase slug flow in nearly horizontal pipes using the multi-fluid model. Int. J. Multiphase Flow, 29 (2003), 1719-1747.

[13] J. Li, Y.Y. Renardy. Direct simulation of unsteady axisymmetric core-annular flow with high viscosity ratio. J. Fluid Mech., 391 (1999), 123-149.

[14] W. Tauber, S.O. Unverdi, G. Tryggvason. The nonlinear behavior of a sheared immiscible fluid interface. Phys. Fluids, 14 (2002), 2871-2885.

[15] T. Boeck, J. Li, E.Y.E. Lopez-Pages, S. Zaleski. Ligament formation in sheared liquid-gas layers. Theor. Comp. Fluid Dyn., 21 (2007), 59-76.

[16] P. Valluri, P.D.M. Spelt, C.J. Lawrence, G.F. Hewitt. Numerical simulation of the onset of slug initiation in laminar horizontal channel flow. Under review for Int. J. Multiphase Flow.

[17] D.S. Lowenherz, C.J. Lawrence. The effect of viscosity stratification on the stability of a free surface flow at low Reynolds numbers. Phys. Fluids A, 1 (1989), 1686-1693.

[18] I.L. Kliakhandler. Long interfacial waves in multilayer thin films and coupled KuramotoSivashinsky equations. J. Fluid Mech., 391 (1999), 45-65.

[19] B.S. Tilley, S.H. Davis, S.G. Bankoff. Nonlinear long-wave stability of superposed fluids in an inclined channel. J. Fluid Mech., 277 (1994), 55-83. 
[20] B.S. Tilley, S.H. Davis, S.G. Bankoff. Linear stability theory of two-layer fluid flow in an inclined channel. Phys. Fluids A, 6 (1994), 3906-3922.

[21] T.M. Segin, B.S. Tilley, L. Kondic. On undercompressive shocks and flooding in countercurrent two-layer flows. J. Fluid Mech., 532 (2005), 217-242.

[22] T.M. Segin, B.S. Tilley, L. Kondic. Long-wave linear stability theory for two-fluid channel flow including compressibility effects. IMA J. Appl. Math. 71 (2006), 715-739.

[23] O.K. Matar, C.J. Lawrence, G.M. Sisoev. Interfacial dynamics in pressure-driven two-layer laminar channel flow with high viscosity ratios. Phys. Rev. E., 75 (2007), 056314, 1-12.

[24] A.L. Bertozzi, M.P. Brenner. Linear stability and transient growth in driven contact lines. Phys. Fluids, 9 (1997), 530-539.

[25] M.H. Eres, L.W. Schwartz, R.V. Roy. Fingering phenomena for driven coating films. Phys. Fluids, 12(6) (2000), 1278-1295.

[26] V.Ya. Shkadov. 1967 Wave flow regimes of a thin layer of viscous fluid subject to gravity. Fluid Dyn., 2 (1967), 21-34.

[27] V. Ya. Shkadov, G.M. Sisoev. Numerical bifurcation analysis of the travelling waves on a falling liquid film. Comp. Fluids, 34 (2005), 151-168. 